\title{
First trimester screening for trisomy 18 by a combination of nuchal translucency thickness and epigenetic marker level
}

\author{
Da Eun Lee', Shin Young Kim¹, Hyun Jin Kim¹, So Yeon Park', Min Hyoung Kim², You Jung Han², and Hyun Mee Ryư ${ }^{1,2, *}$ \\ ${ }^{1}$ Laboratory of Medical Genetics, Medical Research Institute, Cheil General Hospital and Women's Healthcare Center, Seoul, Korea \\ ${ }^{2}$ Department of Obstetrics and Gynecology, Cheil General Hospital and Women's Healthcare Center, Dankook University College of Medicine, \\ Seoul, Korea
}

\begin{abstract}
Purpose: The aim of this study was to assess the diagnostic efficacy of noninvasive prenatal screening for trisomy 18 by assessing the levels of unmethylated-maspin (U-maspin) and fetal nuchal translucency (NT) thickness during the first trimester of pregnancy.

Materials and Methods: A nested case-control study was conducted using maternal plasma samples collected from 65 pregnant women carrying 11 fetuses with trisomy 18 and 54 normal fetuses. We compared the U-maspin levels, NT thicknesses, or a combination of both in the first trimester between the case and control groups.

Results: U-maspin levels and NT thickness were significantly elevated in the first trimester in pregnant women carrying fetuses with trisomy 18 when compared to those carrying normal fetuses $(27.2$ vs. 6.6 copies $/ \mathrm{mL}, P<0.001$ for U-maspin; 5.9 vs. 2.0 $\mathrm{mm}, P<0.001$ for NT). The sensitivities of the U-maspin levels and NT thickness in prenatal screening for fetal trisomy 18 were $90.9 \%$ and $90.9 \%$, respectively, with a specificity of $98.1 \%$. The combined U-maspin levels and NT thickness had a sensitivity of $100 \%$ in prenatal screening for fetal trisomy 18 , with a specificity of $98.1 \%$.

Conclusion: A combination of U-maspin levels and NT thickness is highly efficacious for noninvasive prenatal screening of fetal trisomy 18 in the first trimester of pregnancy.
\end{abstract}

Key words: Trisomy 18, First trimester combined screening, Noninvasive prenatal testing, Epigenetic marker, Nuchal translucency.

\section{Introduction}

Trisomy 18 is caused by the presence of an extra copy of all or part of chromosome 18 , and is the second most common autosomal trisomy with a prevalence of 1 in 6,000 pregnancies. It represents one-third of the chromosomal trisomies identified at 10 to 14 weeks of gestation [1]. Trisomy 18 is associated with severe disability and compromised survival, with at most $10 \%$ of the patients surviving to one year of age. Conventional screening for trisomy 18 has a detection rate of $92 \%$ with a false positive rate (FPR) of $2.1 \%$ [2]. However, a delay in screening until the second trimester means that decisions about invasive testing or therapeutic abortion of pregnancy must be deferred accordingly.

\footnotetext{
Received: 8 May 2017, Revised: 14 June 2017, Accepted: 14 June 2017, Published: 30 June 2017

*Corresponding author: Hyun Mee Ryu, M.D., Ph.D.

Department of Obstetrics and Gynecology, Cheil General Hospital and Women's Healthcare Center, Dankook University College of Medicine, 23 Toegyero 46-gil, Jung-gu, Seoul 04619, Korea.

Tel: +82-2-2000-7175, Fax: +82-2-2278-4574, E-mail: hmryu@yahoo.com

Lee DE and Kim SY contributed equally to this work as co-first authors.

Conflict of interest: The authors declare that they do not have any conflicts of interest.

(c) This is an open-access article distributed under the terms of the Creative Commons Attribution Non-Commercial License (http://creativecommons.org/licenses/by-nc/4.0/) which permits unrestricted non-commercial use, distribution, and reproduction in any medium, provided the original work is properly cited.

(c) Copyright 2017 by the Korean Society of Medical Genetics and Genomics 
In prenatal screening, the fetal nuchal translucency (NT) thickness is the single most effective marker of trisomy 18 and other major chromosomal abnormalities. It appears as a subcutaneous accumulation of fluid behind the fetal neck in a sonogram. NT increases with gestational age, and the incidence of chromosomal abnormalities is related to increasing NT [3]. Increased NT is caused by cardiac failure secondary to structural malformations, abnormalities in the extracellular matrix, abnormal or delayed development of the lymphatic system, and fetal anemia or hypoproteinemia [4-7]. During the first trimester of pregnancy, NT has been widely used as a screening test for fetal aneuploidy either alone or in combination with serum markers [8]. The sensitivity of fetal NT measurements in detecting trisomy 18 is reported to range from $75 \%$ to $91 \%[9,10]$.

Recently, the quantification of fetal DNA by methylationbased DNA discrimination in maternal plasma has been used for noninvasive detection of fetal chromosomal aneuploidies, and promising results have been reported [11-13]. The gene maspin (serpin peptidase inhibitor, clade B [ovalbumin], member 5; SER$P$ INB5) was first identified as a fetal-specific epigenetic marker in maternal plasma that allowed for the measurement of cellfree fetal DNA concentrations in pregnancy-associated disorders [14]. Furthermore, maspin is located on chromosome 18q21.33 and is hypomethylated in the placental (so-called, fetal) cell-free DNA, but completely methylated in the maternal component in cell-free DNA. The quantitative assessment of the placental unmethylated form, unmethylated-maspin (U-maspin) was applied to detect the overrepresentation of chromosome 18 in maternal plasma contributed by a trisomy 18 fetus [15].

The objective of this study was to evaluate the feasibility of prenatal screening for trisomy 18 using a combination of $\mathrm{U}$ maspin levels and fetal NT thickness in the first trimester of pregnancy.

\section{Materials and Methods}

\section{Ethics statement}

This study was conducted in accordance with the Declaration of Helsinki. Appropriate institutional review board approval was obtained for this study from the Ethics Committee at Cheil General Hospital (CGH-IRB-2008-07). Written informed consent was obtained from each participant before the collection of blood samples and subsequent analysis.

\section{Participant recruitment and sample collection}

We performed a nested case-control study of women who enrolled in the Cheil General Hospital Noninvasive Prenatal Testing program. Participants were women who received prenatal care at Cheil General Hospital. For this study, 65 women were selected from a larger sample population of 793 enrolled women, according to the criteria described below. The case group consisted of 11 women who were subsequently assessed via chorionic villus sampling for fetal karyotyping to be carrying a fetus with trisomy 18 . The control group contained 54 women who delivered normal healthy neonates at term (37 weeks of gestation or more) without medical or obstetric complications. The data were collected from pregnant women with singleton pregnancies in whom fetal ultrasound was performed and the fetal NT thickness was measured as part of routine antenatal care at 11 to 14 weeks of gestation. The gestational age was calculated from the first day of the last menstrual period and was used to age-match the trisomy 18 and normal groups. Maternal peripheral blood samples were collected just before chorionic villus sampling in the first trimester.

\section{Sample processing}

Maternal peripheral blood samples $(10 \mathrm{~mL})$ were collected in EDTA tubes (Sarstedt, Nümbrecht, Germany) and centrifuged at $1,600 \times g$ for 10 minutes at $4^{\circ} \mathrm{C}$. The supernatant plasma was re-centrifuged at $16,000 \times g$ for 10 minutes to minimize the additional release of maternal DNA and then stored at $-80^{\circ} \mathrm{C}$ until examined. Circulating fetal DNA was extracted from $1 \mathrm{~mL}$ of maternal plasma using a OIAamp DSP Virus Kit (Qiagen, Hilden, Germany) according to the manufacturer's recommendations. Each DNA sample was eluted into $30 \mu \mathrm{L}$ of sterile DNase-free water and then coded for subsequent blinded analysis.

\section{Real-time quantitative methylation-specific PCR (qMSP) analysis of $\mathrm{U}$-maspin}

Concentrations of U-maspin sequences (GenBank accession no. NT_025028.14) were measured by qMSP assays using a CFX96 Real Time System (Bio-Rad, Hercules, CA, USA). DNA extracted from $1 \mathrm{~mL}$ of maternal plasma was bisulfiteconverted using the EZ DNA Methylation Conversion Kit (Zymo Research, Irvine, CA, USA) according to the manufacturer's protocol. The following U-maspin primers and hydrolysis probe were used for the qMSP assay: forward primer, $5^{\prime}$-TGGTITGTGTGGGTGAGAG-3'; reverse primer, 5'-GGCCGGACTATAAATTACATACATACA-3'; probe, 5'-(VIC) ATTGTTGTATGTATGTTTG (MGBNFQ)-3'. The 5 ' end of the reverse primer was modified by the addition of GGCCGG to enhance the accuracy of the qMSP assays. The PCR reaction mixture contained $12.5 \mathrm{~L}$ of iQ Super- 
mix (Bio-Rad), 200 nM primers, 400 nM hydrolysis probe, and $5 \mathrm{~L}$ of converted DNA in a total volume of $25 \mathrm{~L}$. Initial denaturation cycling conditions were $95^{\circ} \mathrm{C}$ for 10 minutes, followed by 50 cycles of $95^{\circ} \mathrm{C}$ for 15 secocns, $58^{\circ} \mathrm{C}$ for 30 seconds, and $72^{\circ} \mathrm{C}$ for 30 seconds. Calibration curves were prepared for each assay using serial dilutions of single-stranded synthetic DNA oligonucleotides specific to the U-maspin amplicon (Applied Biosystems, Foster City, CA, USA). Each standard was amplified in triplicate and was included on every PCR plate. For U-maspin, the slope and $r^{2}$ values from the standard curves were -3.367 and 0.994 , respectively. The PCR efficiency was calculated from the slope of the curve using the following formula: Efficiency $=10^{(-1 / \text { slope }}-1$. The U-maspin assay amplified with almost optimal efficiency of 98\%.

The U-maspin levels were expressed as copies/mL, and a conversion factor of $6.6 \mathrm{pg}$ of DNA per cell was used in order to express the results as a copy number [16]. Strict precautions were taken against contamination, and multiple negative-control water blanks were included in every analysis. To reduce interexperimental variations, each sample was analyzed in triplicate. The final data reflect the average of the results. The average intra-assay coefficient of variability was 2.1\% for U-maspin.

\section{Fetal NT measurement}

Fetal NT thickness was measured according to the Fetal Medicine Foundation guidelines [17]. The fetus was imaged in the midsagittal plane at a neutral position. The image was magnified so that only the fetal head and upper thorax were included. NT was defined as the normal subcutaneous fluid-filled space between the back of the fetal neck and the overlying skin. The widest thickness of translucency was measured, and the measurements were taken using the inner horizontal line borders of the calipers that were placed on the line that defines NT thickness. Increased NT was defined as the presence of a zone with at least $2.8 \mathrm{~mm}$ of translucency.

Table 1. Clinical characteristics of the case and control groups

\begin{tabular}{lcc}
\multicolumn{1}{c}{ Characteristic } & Trisomy $18(\mathrm{n}=11)$ & Normal $(\mathrm{n}=54)$ \\
\hline Maternal age $(\mathrm{yr})$ & $37(34-40)^{\star}$ & $32(30-34)$ \\
$\geq 35 \mathrm{yr}$ & $8(72.7)$ & $11(20.4)$ \\
Gestational age $(\mathrm{wk})$ & $12.3(12.2-12.4)$ & $12.4(12.1-12.5)$ \\
Body mass index $\left(\mathrm{kg} / \mathrm{m}^{2}\right)$ & $21.4(19.2-21.8)$ & $20.4(18.9-22.2)$ \\
Nulliparity & $4(36.4)$ & $19(35.2)$ \\
Gravidity & $2.0(1.0-3.0)$ & $2.0(1.0-3.0)$ \\
\hline
\end{tabular}

Values are presented as median (interquartile range) or number (\%). ${ }^{*} P<0.01$.

\section{Statistical analysis}

The clinical characteristics of the study population were compared between cases and controls using the Student's ttest for continuous variables and the chi-square test or Fisher's exact test for categorical variables. A comparison of U-maspin levels and NT between the two groups was performed using the Mann-Whitney $U$ test. The combined analysis of $U$-maspin and NT was scored positive if U-maspin or NT was greater than a cutoff value, and was scored negative if U-maspin and NT were less than a cutoff value. The accuracy for detecting fetal trisomy 18 using the U-maspin levels and NT was determined using the results of fetal karyotyping. A receiver operating characteristic (ROC) curve analysis was performed to determine the optimal cutoff value of the factors used for detecting fetal trisomy 18 . The area under the ROC curve (AUC) represents the overall discriminatory ability of a test. The sensitivity, specificity, positive predictive value (PPV), negative predictive value (NPV), FPR, false negative rate (FNR), and accuracy were calculated in accordance with standard formulas. A $P$-value less than 0.05 was considered statistically significant. Statistical analysis was performed using the Statistical Package for Social Sciences version 12.0 (SPSS, Chicago, IL, USA).

\section{Results}

The clinical characteristics of the study groups are summarized in Table 1. At the time of maternal blood sampling, the maternal age was significantly higher in the case group than in the control group $(P<0.01)$. Gravidity, nulliparity, body mass index, and gestational age did not differ significantly between the study groups ( $P>0.05$ for all).

The median concentrations of U-maspin were 27.2 (interquartile range [IR], 23.0-40.6) and 6.6 (IR, 5.3-10.8) copies $/ \mathrm{mL}$ in the case and normal groups, respectively. The concentrations of U-maspin were significantly higher in the case group than in the control group $(P<0.001)$ (Fig. 1). The median NT was $5.9\left(\mathrm{IR}_{\text {, }}\right.$ 5.0-6.7) and $2.0(I R, 1.8-2.2) \mathrm{mm}$ in the case and control groups, respectively. NT was significantly elevated in women carrying fetuses with trisomy 18 when compared to those carrying normal fetuses $(P<0.001)$.

The accuracy of each factor for noninvasive detection of fetal trisomy 18 during the first trimester is shown in Table 2. The optimal cutoff value (19.19) for U-maspin and NT was determined at $90.9 \%$ sensitivity and $98.1 \%$ specificity. The ROC curves of each factor are presented in Fig. 2. The AUCs of U-maspin levels, NT thickness, and a combination of both were 0.985 (95\% con- 
fidence interval [Cl], 0.918-1.000), 0.909 (95\% Cl, 0.812-0.966), and 0.991 (95\% Cl, 0.928-1.000), respectively $(P<0.001$ for all). The sensitivities of $\mathrm{U}$-maspin levels and NT thickness in prenatal screening for fetal trisomy 18 were $90.9 \%$ and $90.9 \%$, respectively, with a specificity of $98.1 \%$ (Table 2). A combined analysis of U-maspin and NT thickness had a sensitivity of 100\% in prenatal screening for fetal trisomy 18 with a specificity of $98.1 \%$, indicating a higher sensitivity for a combined analysis of $\mathrm{U}$ maspin and NT thickness than either alone. The FPR and FNR of the combined analysis were $1.9 \%$ and $0 \%$, respectively, whereas those for U-maspin levels alone were $1.9 \%$ and $0 \%$, respectively, and those for NT thickness alone were 1.9\% and 9.1\%, respectively. Moreover, the PPV and NPV of a combined analysis with U-maspin levels and NT thickness for the detection of trisomy

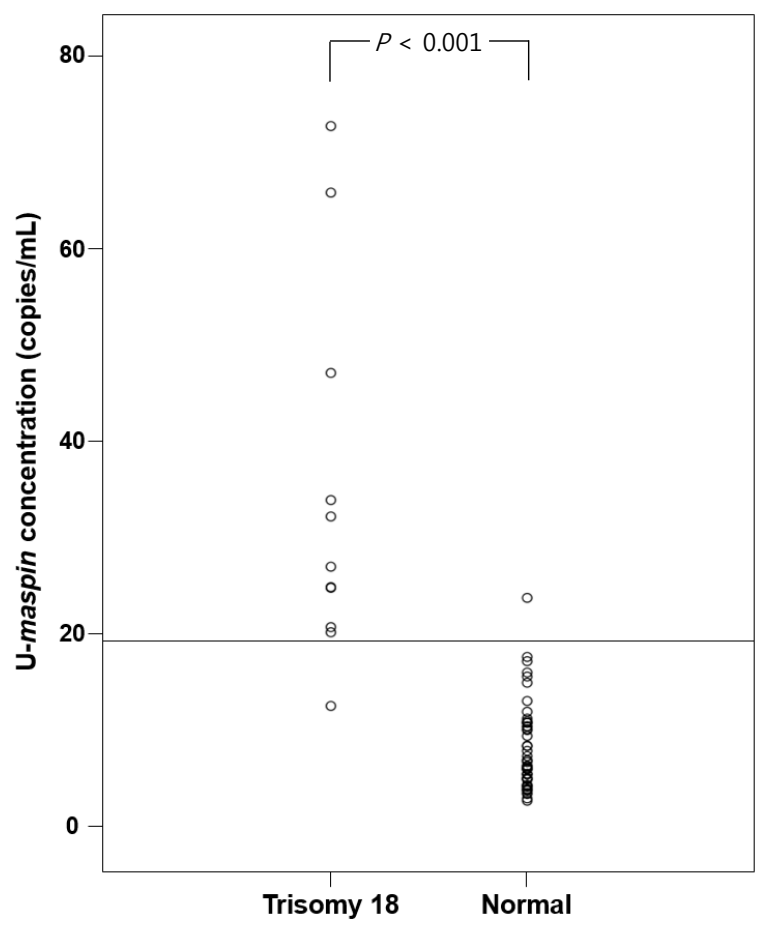

Fig. 1. Unmethylated-maspin (U-maspin) levels in the first trimester maternal plasma from cases and controls. The line represents the optimal cutoff value of U-maspin for the noninvasive detection of fetal trisomy 18.
18 were $91.7 \%$ and $100 \%$, respectively, whereas those of Umaspin alone were $90.9 \%$ and $98.1 \%$, respectively, and those for NT alone were $90.9 \%$ and $98.1 \%$, respectively.

In addition, we observed comparable accuracies using the combined approach according to maternal age (Table 2). Among pregnant women under 35 years of age, the combined analysis of U-maspin levels and NT thickness identified 100\% of fetuses with trisomy $18(n=3)$, with an FPR of 2.3\%. Among pregnant women 35 years of age or older, the combined analysis of $\mathrm{U}$ maspin levels and NT thickness correctly identified both the control $(n=11)$ and case groups $(n=8)$ with a specificity of $100 \%$ and sensitivity of $100 \%$.

\section{Discussion}

This study demonstrated the feasibility of combined testing using U-maspin levels as a fetal-specific epigenetic marker and NT thickness as an ultrasound marker in prenatal testing for

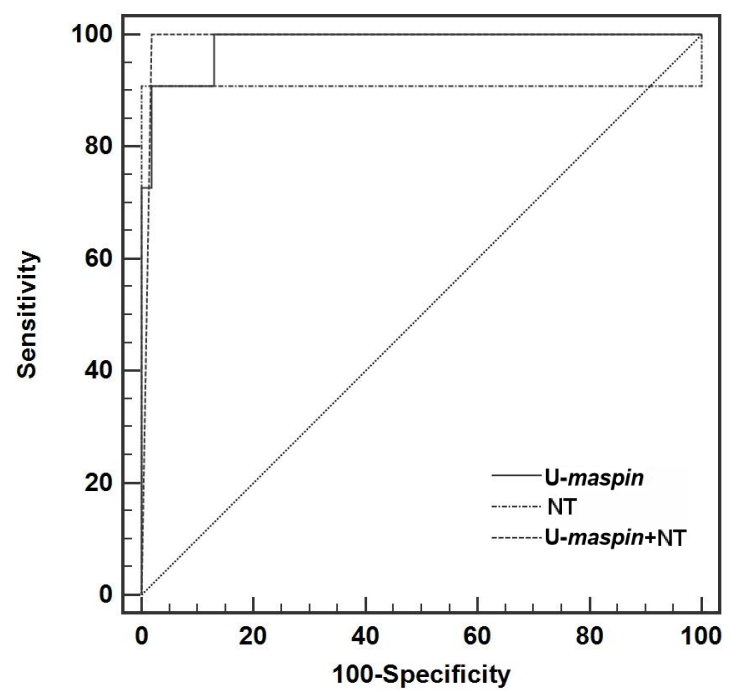

Fig. 2. Receiver operating characteristic curves of unmethylatedmaspin concentration levels (U-maspin), nuchal translucency thickness (NT), and a combination of both (U-maspin+NT), which are represented by solid, dash-dotted, and dashed lines, respectively.

Table 2. Utility of measurements in the detection of trisomy 18

\begin{tabular}{lcccccccc|}
\hline \multicolumn{1}{c}{ Measurement } & Cutoff & Sensitivity (\%) & Specificity (\%) & PPV (\%) & NPV (\%) & FPR (\%) & FNR (\%) & ACC (\%) \\
\hline NT (mm) & 2.8 & 90.9 & 98.1 & 90.9 & 98.1 & 1.9 & 9.1 & 96.9 \\
U-maspin (copies/mL) & 19.19 & 90.9 & 98.1 & 90.9 & 98.1 & 1.9 & 9.1 & 96.9 \\
\hline NT+U-maspin & & 100 & 98.1 & 91.7 & 100 & 1.9 & 0.0 & 98.5 \\
\hline Maternal age $<35$ yr & & 100 & 97.7 & 75.0 & 100 & 2.3 & 0.0 & 97.8 \\
\hline Maternal age $\geq 35 \mathrm{yr}$ & & 100 & 100 & 100 & 100 & 0.0 & 0.0 & 100 \\
\hline
\end{tabular}

PPV, positive predictive value; NPV, negative predictive value; FPR, false positive rate; FNR, false negative rate; ACC, accuracy. 
trisomy 18 during the first-trimester. We found that U-maspin levels and NT thickness were significantly elevated during the first trimester in pregnant women carrying fetuses with trisomy 18 when compared to those carrying normal fetuses. Furthermore, a combination of U-maspin levels and NT thickness correctly identified all pregnant women carrying fetuses with trisomy 18. Among pregnant women aged 35 years or older, this combined approach resulted in perfect classification of all pregnant women with normal and fetuses with trisomy 18 , corresponding to $100 \%$ specificity and $100 \%$ sensitivity. Therefore, our results suggest that a combined approach using U-maspin levels and NT thickness may be a useful noninvasive screening method during the first trimester for fetal trisomy 18.

Increased NT thickness in the first trimester has been reported in trisomy 18 and other chromosomal abnormalities as well as many fetal malformations and genetic syndromes $[18,19]$. Sherod et al. [20] reported increased NT thicknesses in about $75 \%$ of fetuses with trisomy 18 in the first trimester. In a multicenter study, Snijders et al. [3] reported a detection rate of 74.8\% with a 5\% FPR for trisomy 18 by fetal NT thickness. Tul et al. [21] reported increased NT thickness in 39 out of 50 fetuses (78\%) with trisomy 18 at 10 to 14 weeks of gestation. Recently, Sepulveda et al. [9] reported a 91\% detection rate using NT measurements in first trimester pregnancies complicated by trisomy 18 . In our study, the detection rate of $90.9 \%$ that was achieved by NT thickness alone was similar to the findings of Sepulveda et al. [9] and was higher than that in other reports $[3,20,21]$. The minor case of trisomy 18 revealed a normal NT thickness $(1.1 \mathrm{~mm})$, while U-maspin levels were within the abnormal range (20.46 copies/mL).

In a previous study, Chim et al. [22] demonstrated that maspin is unmethylated in the placenta but hypermethylated in maternal blood cells, and its epigenetic differences could serve as a universal fetal DNA marker. Tong et al. [15] described an epigenetic allelic ratio analysis, first by using bisulfite conversion followed by methylation-specific PCR and primer extension reactions designed to distinguish the $A$ and $C$ alleles of fetusderived U-maspin based on size for the noninvasive prenatal detection of fetal trisomy 18. The test had a sensitivity of 100\% with an FPR of 9.7\% in placental tissues. However, the allelic ratio analysis was informative in only 38 of 187 placental tissue samples tested in the first or third trimester. Moreover, this study did not evaluate the clinical performance of the test in maternal plasma samples obtained in the first trimester. In our approach, U-maspin levels had a sensitivity of $90.9 \%$ in prenatal screening for fetal trisomy 18, with an FPR of 1.9\% in maternal plasma samples. Moreover, the test was informative in all maternal plasma samples obtained during the first trimester (less than 14 weeks of gestation). However, a single case of trisomy 18 was within the normal range (12.9 copies $/ \mathrm{mL}$ ) and showed an abnormal NT thickness $(8.9 \mathrm{~mm})$.

First trimester combined screening for trisomy 18 is based on a combination of maternal age, fetal NT thickness, and maternal serum biochemical markers such as human chorionic gonadotropin (hCG), and pregnancy-associated plasma protein A (PAPP-A), and has a predicted sensitivity of 70 to $90.9 \%$ with an FPR of less than 2\% [23-25]. More recently, integrated testing using combinations of NT measurement and PAPP-A in the first trimester and various maternal serum biochemical markers, including alpha fetoprotein, unconjugated estriol, hCG, and inhibin-A in the second trimester have improved screening performance and achieved a detection rate of $92 \%$ with an FPR of 2.1\% for trisomy 18 [2]. However, delaying screening until the second trimester unnecessarily increases maternal anxiety, as well as the likelihood that bonding with the fetus has already occurred. Moreover, the integrated testing may delay the early decision for invasive testing or therapeutic abortion. Therefore, there is an urgent need for the development of accurate screening strategies in the first trimester.

In the present study, we evaluated the utility and accuracy of noninvasive screening for fetal trisomy 18 using U-maspin levels and NT thickness during the first trimester of pregnancy. Our combined approach allowed detection of 11/11 (100\%) fetuses with trisomy 18 with an FPR of 1.9\%. This approach showed an improved detection rate compared with using NT thickness or U-maspin levels alone. Moreover, among women 35 years of age or older, the combined approach identified 100\% of fetuses with trisomy 18, with an FPR of 0\%. Thus, our results demonstrate that combining U-maspin levels and NT thickness not only is more sensitive than previous combined screening methods, but also allays parental anxiety by providing earlier results about chromosomal abnormalities in the first trimester. Furthermore, this combined approach is a relatively simple noninvasive screening method compared to previous screening tests that use a combination of four or more markers.

To our knowledge, this is the first study using a combination of a fetal-specific epigenetic marker and ultrasound marker analysis with U-maspin levels and NT thickness for noninvasive detection of trisomy 18 during the first trimester. Our findings suggest that this combination is useful in screening for fetal trisomy 18 in the first trimester of pregnancy. However, this study is limited by its small sample size; therefore, the $100 \%$ detection 
rate in this study should be interpreted with caution. Further studies with a larger clinical cohort are required to validate the screening performance of this protocol.

\section{Acknowledgements}

This study was supported by a grant from the Korean Health Technology R\&D Project, Ministry of Health \& Welfare, Republic of Korea (HI13C1934).

We thank the following physicians who contributed to this study; Jin Woo Kim, Do Jin Kim, Bom Yi Lee, Ju Yeon Park, Eun Young Choi, Ji Hyae Lim, Yeon Woo Lee, Ah Rum Oh, Shin Yeong Lee, and So Min Seo.

\section{References}

1. Snijders RJ, Sebire NJ, Nicolaides KH. Maternal age and gestational age-specific risk for chromosomal defects. Fetal Diagn Ther 1995;10:356-67.

2. Bestwick JP, Huttly WJ, Wald NJ. Detection of trisomy 18 and trisomy 13 using first and second trimester Down's syndrome screening markers. J Med Screen 2013;20:57-65.

3. Snijders RJ, Noble P, Sebire N, Souka A, Nicolaides KH. UK multicentre project on assessment of risk of trisomy 21 by maternal age and fetal nuchal-translucency thickness at 10-14 weeks of gestation. Fetal Medicine Foundation First Trimester Screening Group. Lancet 1998;352:343-6.

4. Makrydimas G, Sotiriadis A, loannidis JP. Screening performance of first-trimester nuchal translucency for major cardiac defects: a metaanalysis. Am J Obstet Gynecol 2003;189:1330-5.

5. von Kaisenberg CS, Krenn V, Ludwig M, Nicolaides KH, Brand-Saberi B. Morphological classification of nuchal skin in human fetuses with trisomy 21,18 , and 13 at 12-18 weeks and in a trisomy 16 mouse. Anat Embryol (Berl) 1998;197:105-24.

6. Souka AP, Krampl E, Geerts L, Nicolaides KH. Congenital lymphedema presenting with increased nuchal translucency at 13 weeks of gestation. Prenat Diagn 2002;22:91-2.

7. Tercanli S, Miny P, Siebert MS, Hösli I, Surbek DV, Holzgreve W. Fanconi anemia associated with increased nuchal translucency detected by first-trimester ultrasound. Ultrasound Obstet Gynecol 2001;17:1602.

8. Miron P, Côté YP, Lambert J. Nuchal translucency thresholds in prenatal screening for Down syndrome and trisomy 18. J Obstet Gynaecol Can 2009;31:227-35.

9. Sepulveda $W$, Wong $A E$, Dezerega $V$. First-trimester sonographic findings in trisomy 18: a review of 53 cases. Prenat Diagn 2010;30:256-9.
10. Nicolaides KH. Nuchal translucency and other first-trimester sonographic markers of chromosomal abnormalities. Am J Obstet Gynecol 2004;191:45-67.

11. Tong $Y K_{1}$ Jin $S$, Chiu RW, Ding $C$, Chan KC, Leung TY, et al. Noninvasive prenatal detection of trisomy 21 by an epigenetic-genetic chromosome-dosage approach. Clin Chem 2010;56:90-8.

12. Tsui DW, Lam YM, Lee WS, Leung TY, Lau TK, Lau ET, et al. Systematic identification of placental epigenetic signatures for the noninvasive prenatal detection of Edwards syndrome. PLoS One 2010;5:e15069.

13. Lim JH, Kim SY, Park SY, Lee SY, Kim MJ, Han YJ, et al. Non-invasive epigenetic detection of fetal trisomy 21 in first trimester materna plasma. PLoS One 2011;6:e27709.

14. Dokras A, Gardner LM, Kirschmann DA, Seftor EA, Hendrix MJ. The tumour suppressor gene maspin is differentially regulated in cytotrophoblasts during human placental development. Placenta 2002;23:274-80.

15. Tong YK, Ding C, Chiu RW, Gerovassili A, Chim SS, Leung TY, et al. Noninvasive prenatal detection of fetal trisomy 18 by epigenetic allelic ratio analysis in maternal plasma: theoretical and empirical considerations. Clin Chem 2006;52:2194-202.

16. Lo YM, Tein MS, Lau TK, Haines CJ, Leung TN, Poon PM, et al. Quantitative analysis of fetal DNA in maternal plasma and serum: implications for noninvasive prenatal diagnosis. Am J Hum Genet 1998;62:768-75.

17. The Fetal Medicine Foundation. 11-13 week scan. Nuchal translucency. Protocol for measurement of nuchal translucency. [https://fetalmedicine.org/training-n-certification/certificates-of-competence/ nuchal-translucency-scan]

18. Souka AP, Snijders RJ, Novakov A, Soares W, Nicolaides KH. Defects and syndromes in chromosomally normal fetuses with increased nuchal translucency thickness at 10-14 weeks of gestation. Ultrasound Obstet Gynecol 1998;11:391-400

19. Souka AP, Krampl E, Bakalis S, Heath V, Nicolaides KH. Outcome of pregnancy in chromosomally normal fetuses with increased nuchal translucency in the first trimester. Ultrasound Obstet Gynecol 2001;18:9-17.

20. Sherod C, Sebire NJ, Soares W, Snijders RJ, Nicolaides KH. Prenatal diagnosis of trisomy 18 at the 10-14-week ultrasound scan. Ultrasound Obstet Gynecol 1997;10:387-90.

21. Tul N, Spencer $K_{1}$ Noble $P$, Chan $C$, Nicolaides $K$. Screening for trisomy 18 by fetal nuchal translucency and maternal serum free beta-hCG and PAPP-A at 10-14 weeks of gestation. Prenat Diagn 1999;19:1035-42.

22. Chim SS, Tong YK, Chiu RW, Lau TK, Leung TN, Chan LY, et al. Detection of the placental epigenetic signature of the maspin gene in maternal plasma. Proc Natl Acad Sci U S A 2005;102:14753-8. 
23. Wapner R, Thom E, Simpson JL, Pergament E, Silver R, Filkins $K$, et al. First-trimester screening for trisomies 21 and 18. N Engl J Med 2003;349:1405-13.

24. Wright D, Kagan KO, Molina FS, Gazzoni A, Nicolaides KH. A mixture model of nuchal translucency thickness in screening for chromo- somal defects. Ultrasound Obstet Gynecol 2008;31:376-83.

25. Kagan KO, Wright $D$, Valencia C, Maiz N, Nicolaides KH. Screening for trisomies 21, 18 and 13 by maternal age, fetal nuchal translucency, fetal heart rate, free beta-hCG and pregnancy-associated plasma protein-A. Hum Reprod 2008;23:1968-75. 